\title{
Right hepatectomy due to portal vein thrombosis in vasculobiliary injury following laparoscopic cholecystectomy: a case report
}

\author{
Stipislav Jadrijevic ${ }^{1}$, Davorin Sef ${ }^{{ }^{*}}$, Branislav Kocman ${ }^{1}$, Anna Mrzljak ${ }^{2,4}$, Hrvoje Matasic $^{3}$ and Dinko Skegro ${ }^{2,4}$
}

\begin{abstract}
Introduction: Vasculobiliary injury composed of bile duct, portal vein and hepatic artery injury is a rare, but the most severe, complication after cholecystectomy that may require hepatectomy or even urgent liver transplantation.

Case presentation: We present a case of a 36-year-old Caucasian woman with a biliary sepsis and a large right liver lobe abscess due to an extreme vasculobiliary injury after laparoscopic cholecystectomy. Bismuth type IV biliary duct injury, portal vein thrombosis and injury of right hepatic artery were identified, resulting in life-threatening septic episodes. Right hepatectomy with Roux-en-Y hepaticojejunostomy and reconstruction of her portal vein with a vein allograft were performed. She fully recovered and remained well during 3 years of follow-up.

Conclusions: Although rare, the impact of vasculobiliary injuries after cholecystectomy highlights the need for constant alertness and prompt management in order to minimize the risk of the routine operative procedure. Hepatectomy with biliary and vascular reconstruction should be considered early in the management of vasculobiliary injury to avoid the development of life-threatening consequences.
\end{abstract}

Keywords: Bile duct injury, Hepatectomy, Hepatic artery injury, Laparoscopic cholecystectomy, Portal vein thrombosis, Vasculobiliary injury

\section{Introduction}

Laparoscopic cholecystectomy (LC) is the procedure of choice for symptomatic cholelithiasis, but is associated with a higher incidence of iatrogenic bile duct injuries than the open procedure $[1,2]$. Vasculobiliary injury (VBI), defined as bile duct, hepatic artery and/or portal vein injury, has been recognized as one of the most severe complications after cholecystectomy that may result in various degrees of hepatic ischemia with subsequent liver necrosis, abscess formation, acute liver failure or secondary biliary cirrhosis. [3-5]. Biliary tree anomalies present in up to $25 \%$ of patients [6], and may lead to anatomical misidentification and technical problems that contribute to the development of these injuries. The management of VBI depends considerably on development of biliary ischemia and hepatic infarction, necessitating in some cases hepatectomy or

\footnotetext{
* Correspondence: davorin.sef@gmail.com

'Department of Surgery, Division of Transplantation Surgery, University

Hospital Merkur, Zagreb, Croatia

Full list of author information is available at the end of the article
}

even urgent liver transplantation [7]. However, the best treatment strategy and timing of surgical repair when there is a VBI is still controversial.

Here we present a management of VBI after LC. The presented case emphasizes the need for prompt and adequate management of patients with VBI in order to avoid life-threatening complications.

\section{Case presentation}

A previously healthy 36-year-old Caucasian woman underwent early LC for an acute cholecystitis in an affiliated hospital. Due to uncontrolled bleeding, LC was converted to an open procedure through a standard right subcostal laparotomy. Hemostasis was achieved using sutures and clips, and common bile duct injury was repaired using a T-tube. Postoperatively she became febrile and complained of right upper abdominal pain; a laboratory examination revealed cholestatic profile of liver tests (total bilirubin $99.3 \mu \mathrm{mol} / \mathrm{L}$, alkaline phosphatase $207 \mathrm{IU} / \mathrm{L}$, gamma-glutamyl transferase 209IU/L) and increased 
inflammatory markers (C-reactive protein $129.4 \mathrm{mg} / \mathrm{L}$ ). Secondary cholangiography showed a Bismuth type IV common bile duct transection with biliary leak along the T-tube. Blood cultures tested positive for methicillinresistant Staphylococcus aureus and Acinetobacter baumannii, and parenteral therapy with vancomycin and colistin was initiated. Portal vein thrombosis (Figure 1) and right hepatic artery injury (Figure 2) were identified by hepatic angiography. Four weeks after the LC, the patient developed systemic inflammatory response syndrome (SIRS) and was transferred to our hospital. Abdominal computed tomography confirmed the previous findings, as well as a large right liver lobe abscess accompanied by perihepatic and interintestinal biloma (Figure 3).

The further surgical management included right subcostal laparotomy revealing a diffuse peritonitis with severe inflammatory reaction of her hepatic hilum, a large biloma and right hepatic duct and right hepatic artery suture ligatures as well as portal vein suture ligatures with thrombosis above the splenomesenteric confluence. Based on these findings, right hepatectomy with a left Roux-en-Y hepaticojejunostomy was performed. Due to the distal portal vein thrombosis and portal vein suture ligatures (above the splenomesenteric confluence) reconstruction with cadaveric iliac vein allograft was performed. A 6-dayold vein allograft of identical blood group and anatomical match was used. The postoperative period was further complicated with the biliary sepsis, bilateral pleural effusions and pneumonia that subsequently resolved. She was

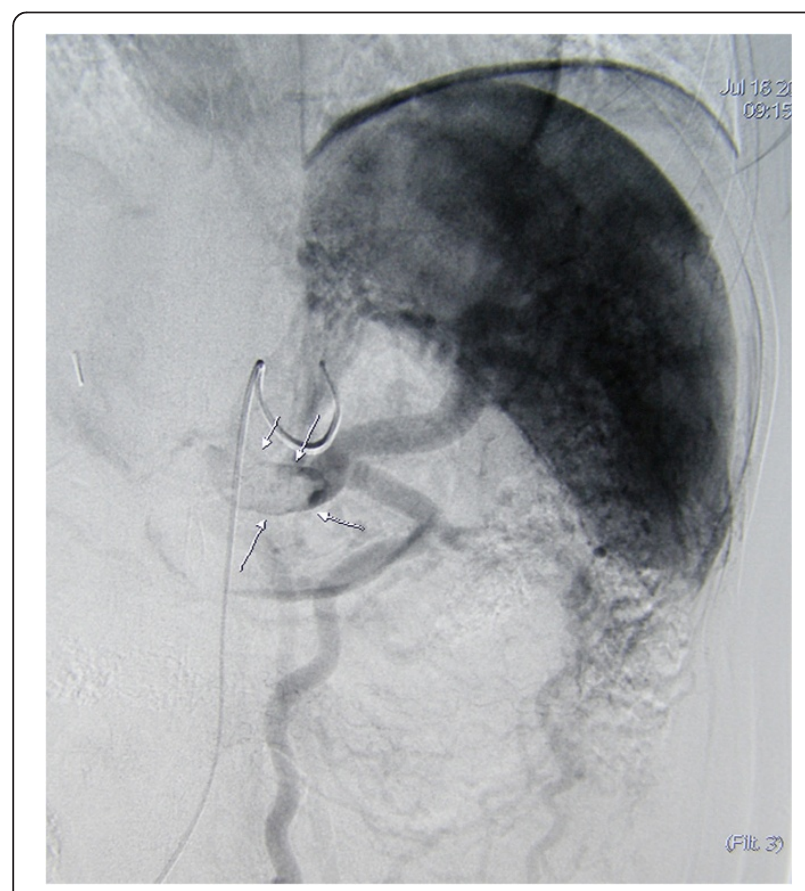

Figure 1 Angiography shows occlusion and thrombosis in the portal vein down to the splenomesenteric confluence (arrows).

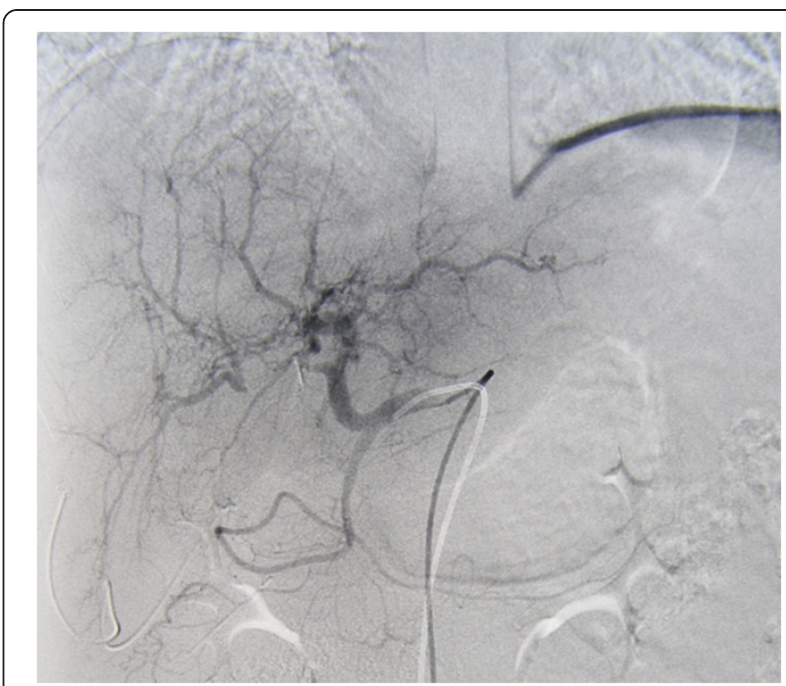

Figure 2 Angiography after laparoscopic cholecystectomy showing occlusion of right hepatic artery.

discharged from the hospital 60 days after the hepatectomy, fully recovered. After 2 years of follow-up, her liver function tests are unremarkable with patent allograft as demonstrated by Doppler ultrasound (Figure 4).

\section{Discussion}

A VBI as an injury of a bile duct and adjacent vascular structures is mainly caused by operative trauma during cholecystectomy. Injuries to the bile duct and liver vessels may occur due to mistakes in dissection, so their identification and careful dissection around the neck of the gallbladder is the best way to avoid them. Common bile duct or aberrant right hepatic ducts are most often

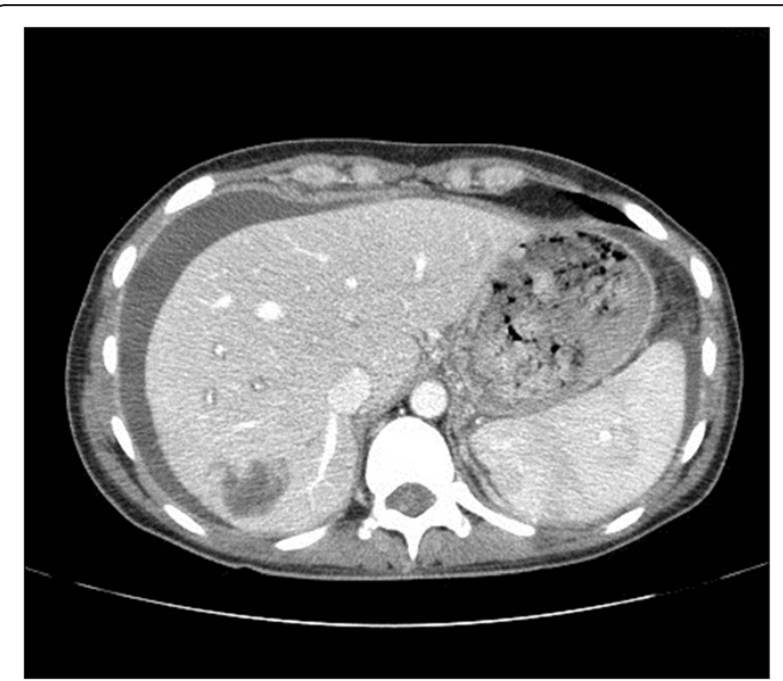

Figure 3 Abdominal computed tomography image showing large right liver lobe abscess accompanied by perihepatic and interintestinal biloma. 


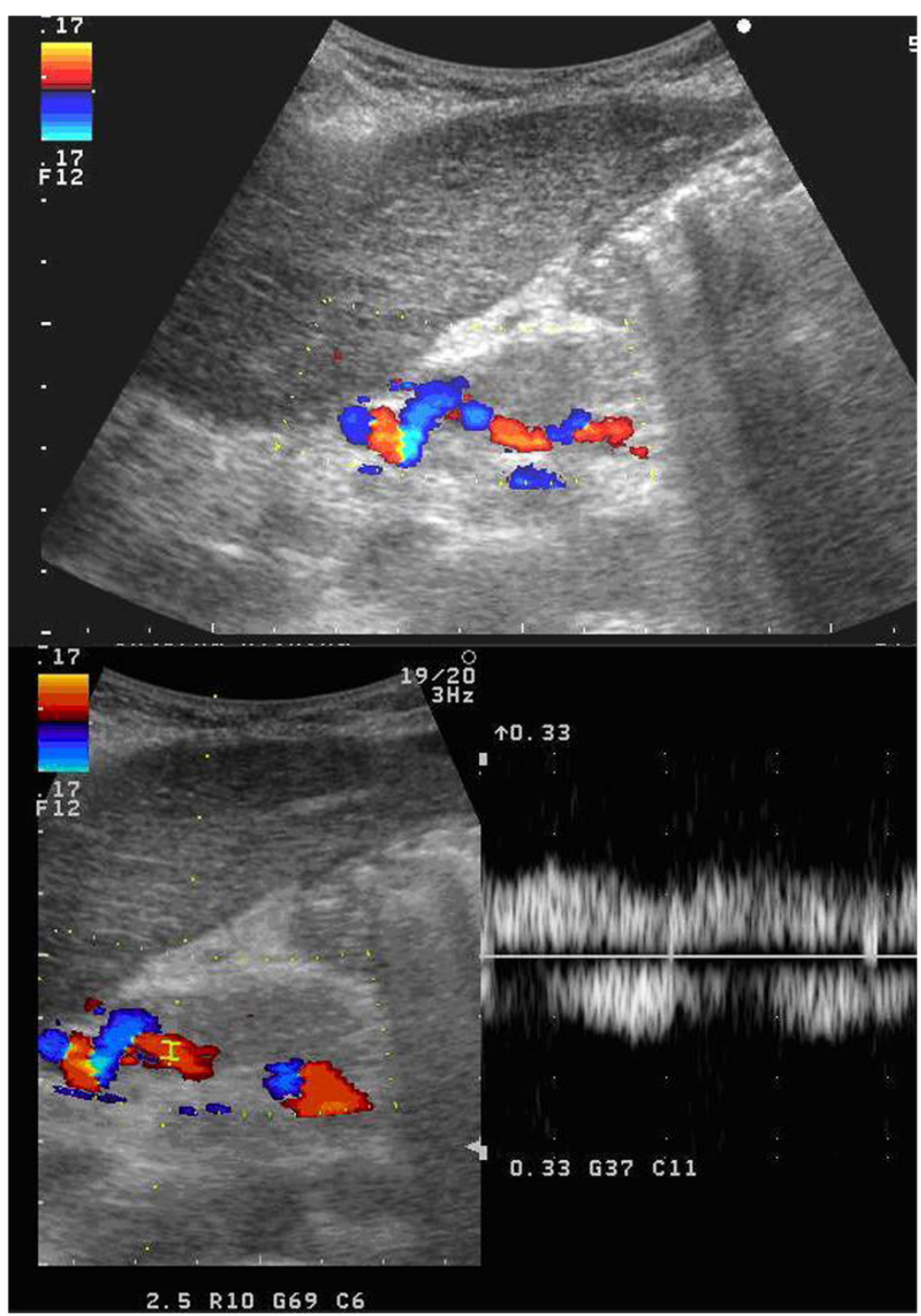

Figure 4 Abdominal Doppler ultrasound 2 years after right hepatectomy with reconstruction of portal vein showing normal liver parenchyma and good graft patency.

misidentified as the cystic duct. The most dangerous biliary anomaly is the cystic duct that runs along the side of a low-lying aberrant right segmental duct. Most commonly this is the right posterior hepatic duct, occurring in 2.5 to $8 \%$ of patients, which drains liver segments 6 and 7 [3]. Therefore, performing meticulous dissection around the gallbladder neck and cystic duct before clip placement enables visualization of any variant anatomy. In up to $92 \%$ of cases, biliary injury is accompanied by injury of the right hepatic artery [8]. In rare cases, the portal vein alone or in combination with other arteries is involved. These data are variable in some reports due to the fact that angiography was mostly performed selectively. Therefore, routine hepatic arteriography is recommended in all patients with biliary duct injury after cholecystectomy if early repair is considered [8]. Further on, since hepatic parenchyma depends on portal circulation in cases of arterial injury, examination of the portal blood flow is necessary in any case of VBI [9].

So far, the data regarding specific type of VBI composed of biliary duct, portal vein and hepatic artery are scarce, especially in terms of management and outcome [8-12]. Portal vein resistance to injury or under-reporting of such cases due to rapid clinical deterioration and death may be some of the reasons [8]. Portal vein thrombosis in VBI is in the majority of cases accompanied by injury of a major hepatic artery, predominantly the right hepatic artery [8]. This type of VBI, compromising dual hepatic supply, results in more rapid and severe hepatic ischemia compared to isolated arterial injury. The treatment of this potentially lethal complication might require liver resection or even liver transplantation $[8-11,13,14]$. Unrecognized VBI in 
patients who remain asymptomatic can lead to development of biliary strictures, cholangitis and liver atrophy [14].

Current data demonstrate that rapid liver necrosis in almost half of patients with VBI with dual vascular injury resulted in right hepatectomy within the first 2 weeks after the injury with a mortality rate of $50 \%$ [8]. In our patient, uncontrolled clipping and suturing during the cholecystectomy resulted in right biliary duct and hepatic artery injury with subsequent portal vein thrombosis. Right hepatectomy was performed 4 weeks after the development of VBI, however, in a critically ill patient SIRS developed due to biliary sepsis and postponed treatment of the liver abscess. In our case, portal vein resection and reconstruction using a vein graft had to be performed due to partial iatrogenic injury and thrombosis of the portal vein. The prompt management and early referral to a tertiary center is therefore of paramount importance since the consequences of rapid hepatic ischemia in this type of injury may be devastating.

Liver transplantation is still a controversial option for patients with complex VBI, mainly because of concomitant septic complications and low survival rates, although recently it has been reported in the treatment of long-term complications such as secondary biliary sclerosis [7].

\section{Conclusions}

Considering the reported rarity on the one hand and its devastating consequences on the other hand, it is important to compile the evidence and experience regarding portal vein thrombosis in VBI, in order to establish proper management strategies and improve patients' outcomes. Therefore, the present case contributes to the emerging literature about this issue emphasizing the need for the prompt management and early referral to a tertiary center where hepatic resection with biliary and vascular reconstruction can be safely performed.

\section{Consent}

Written informed consent was obtained from the patient for publication of this case report and accompanying images. A copy of the written consent is available for review by the Editor-in-Chief of this journal.

\section{Abbreviations \\ LC: Laparoscopic cholecystectomy; SIRS: Systemic inflammatory response syndrome; VBI: Vasculobiliary injury}

\section{Competing interests}

The authors declare that they have no competing interests.

\section{Authors' contributions}

SJ was a major contributor in the writing of this manuscript. SJ, BK, DaS and HM performed the surgery and SJ and DaS analyzed the data. DiS performed the ultrasound examination of the patient. SJ, DaS, BK, AM, HM, and DiS provided important intellectual content and helped revise the manuscript. All authors read and approved the final manuscript.

\section{Acknowledgements}

A special acknowledgement goes to the patient on whom the case report is based. The authors acknowledge the help of the operating room, intensive care and department personnel who assisted in the care of this patient.

\section{Author details}

'Department of Surgery, Division of Transplantation Surgery, University Hospital Merkur, Zagreb, Croatia. ${ }^{2}$ Department of Medicine, University Hospital Merkur, Zagreb, Croatia. ${ }^{3}$ Department of Anesthesiology and Critical Care, University Hospital Merkur, Zagreb, Croatia. ${ }^{4}$ School of Medicine, University of Zagreb, Zagreb, Croatia.

Received: 25 May 2014 Accepted: 16 September 2014 Published: 7 December 2014

\section{References}

1. Csikesz NG, Tseng JF, Shah SA: Trends in surgical management for acute cholecystitis. Surgery 2008, 144(2):283-289.

2. Manouras A, Pararas N, Antonakis P, Lagoudiannakis EE, Papageorgiou G, Dalianoudis IG, Konstadoulakis MM: Management of major bile duct injury after laparoscopic cholecystectomy: a case report. J Med Case Rep 2009, 3:44.

3. Caminiti R, Rossitto M, Ciccolo A: Pseudoaneurysm of the hepatic artery and hemobilia: a rare complication of laparoscopic cholecystectomy; clinical case and literature review. Acta Chir Belg 2011, 111(6):400-403.

4. Alves A, Farges O, Nicolet J, Watrin T, Sauvanet A, Belghiti J: Incidence and consequence of an hepatic artery injury in patients with postcholecystectomy bile duct strictures. Ann Surg 2003, 238:93-96.

5. Buell JF, Cronin DC, Funaki B, Koffron A, Yoshida A, Lo A, Leef J, Millis JM: Devastating and fatal complications associated with combined vascular and bile duct injuries during cholecystectomy. Arch Surg 2002, 137:703-710

6. Babel N, Sakpal SV, Paragi P, Wellen J, Feldman S, Chamberlain RS: latrogenic bile duct injury associated with anomalies of the right hepatic sectoral ducts: a misunderstood and underappreciated problem. HPB Surg 2009, 2009:153269.

7. Fernández JA, Robles R, Marín C, Sánchez-Bueno F, Ramírez P, Parrilla P: Laparoscopic iatrogeny of the hepatic hilum as an indication for liver transplantation. Liver Transp/ 2004, 10(1):147-152.

8. Strasberg SM, Helton WS: An analytical review of vasculobiliary injury in laparoscopic and open cholecystectomy. HPB (Oxford) 2011, 13(1):1-14.

9. Stewart L, Robinson TN, Lee CM, Liu K, Whang K, Way LW: Right hepatic artery injury associated with laparoscopic bile duct injury: incidence, mechanism, and consequences. J Gastrointest Surg 2004, 8:523-530

10. Laurent A, Sauvanet A, Farges O, Watrin T, Rivkine E, Belghiti J: Majo hepatectomy for the treatment of complex bile duct injury. Ann Surg 2008, 248(1):77-83.

11. Thomson BN, Parks RW, Madhavan KK, Garden OJ: Liver resection and transplantation in the management of iatrogenic biliary injury. World J Surg 2007, 31(12):2363-2369.

12. Ragozzino A, Lassandro F, De Ritis $R$, Imbriaco M: Value of MRI in three patients with major vascular injuries after laparoscopic cholecystectomy. Emerg Radiol 2007, 14(6):443-447.

13. Schmidt SC, Langrehr JM, Raakow R, Klupp J, Steinmüller T, Neuhaus $P$. Right hepatic lobectomy for recurrent cholangitis after combined bile duct and right hepatic artery injury during laparoscopic cholecystectomy: a report of two cases. Langenbecks Arch Surg 2002. 387:183-187.

14. Heinrich S, Seifert $H$, Krahenbuhl L, Fellbaum C, Lorenz M: Right hemihepatectomy for bile duct injury following laparoscopic cholecystectomy. Surg Endosc 2003, 17:1494-1495.

doi:10.1186/1752-1947-8-412

Cite this article as: Jadrijevic et al.: Right hepatectomy due to portal vein thrombosis in vasculobiliary injury following laparoscopic cholecystectomy: a case report. Journal of Medical Case Reports 2014 8:412. 